\title{
Gateway Placement for Throughput Optimization in Wireless Mesh Networks
}

\author{
Fan Li Yu Wang \\ Department of Computer Science \\ University of North Carolina at Charlotte, USA \\ Email: \{fli, ywang32\}@uncc.edu
}

\author{
Xiang-Yang Li \\ Department of Computer Science \\ Illinois Institute of Technology, USA \\ Email: xli@cs.iit.edu
}

\begin{abstract}
In this paper, we address the problem of gateway placement for throughput optimization in multi-hop wireless mesh networks. Assume that each mesh nodes in the mesh network has a traffic demand. Given the number of gateways to deploy (denoted by $k$ ) and the interference model in the network, we study how to place exactly $k$ gateways in the mesh network such that the total throughput that can be supported is maximized while it also ensure a certain fairness among all mesh nodes. We propose a novel grid-based gateway deployment method using a cross-layer throughput optimization. Our proposed method can also be extended to work with multi-channel and multi-radio mesh networks. Our extensive evaluation demonstrates that our method can effectively exploit the resources available and it performs much better than random deployment and fixed deployment methods.
\end{abstract}

\section{INTRODUCTION}

Wireless mesh network (WMN) [1] draws lots of attentions in recent years due to its various potential applications, such as broadband home networking, community and neighborhood networks, and enterprise networking. It has been used as the last mile solution for extending the Internet connectivity for mobile nodes. Many US cities (e.g., Medford, Oregon; Chaska, Minnesota; and Gilbert, Arizona) have already deployed mesh networks. AWA, the Spanish operator of Wireless LAN networks, will roll out commercial WLAN and mesh networks for voice and data services. Several companies such as MeshDynamics have recently announced the availability of multi-hop multi-radio mesh network technology. These networks behave almost like wired networks since they have infrequent topology changes, limited node failures, etc.. For wireless mesh networks, the aggregate traffic load of each routing node changes infrequently also. A unique characteristic of wireless networks is that the communication channels are shared by the wireless terminals. Thus, one of the major problems facing wireless networks is the reduction of capacity due to interference caused by simultaneous transmissions. Using multiple channels and multiple radios can alleviate but not eliminate the interference.

Wireless mesh networks consist of two types of nodes: mesh routers and mesh clients. Mesh routers form an infrastructure (called mesh backbone) for mesh clients that connect to them. The mesh backbone can be built using various types of radio technologies. The mesh routers form a mesh of self-configuring, self-healing links among themselves.
Compared with a conventional wireless routers, mesh routers can achieve the same coverage with much lower transmission power through multi-hop communication. To connect the mesh network to the Internet, gateway devices are needed. Usually, in mesh networks some mesh routers have the gateway functionality which can provide the connectivity to the Internet. The common network infrastructure for mesh networks is illustrated in Figure 1, where dash and solid lines indicate wireless and wired links. We do not include the mesh clients in the figure, since this paper focuses on the design of the mesh backbone only. Hereafter, we will call the mesh routers without gateway functionality mesh nodes or just mesh routers, and call the mesh routers with functionality gateway nodes to distinguish them from mesh nodes.

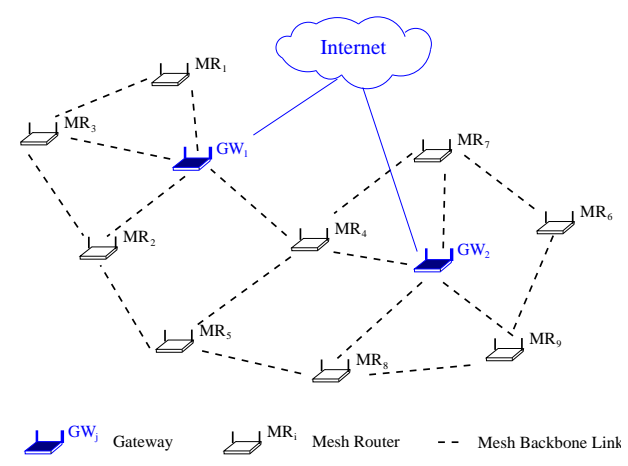

Fig. 1. Illustration of network infrastructure of a wireless mesh network.

In this paper, we will study how to design the mesh backbone to optimize the network throughput under the inference. More specifically, given the mesh backbone and the number of gateway devices, we will study how to place the gateway devices in the mesh backbone in order to achieve optimal throughput. The application scenario of this gateway deployment problem for a community networks is as follows. The mesh routers are placed on the roof of houses in a neighborhood, which serve as access points for users inside the homes and along the roads. All these mesh routers are fixed and form the mesh network. Then the mesh service provider need to decide where to put the gateway devices to connect the mesh network to the Internet. Since different gateway deployment causes different mesh backbone topologies and affects the network throughput, it is important to find optimal 
gateway deployment to maximize the throughput.

Optimal the throughput has been studied in wireless networks. Gupta and Kumar [2] studied the asymptotic capacity of a multi-hop wireless networks. Recently, several papers [3], [4] further studied the capacity of wireless networks under different models. Kyasanur and Vaidya [5] studied the capacity region on random multi-hop multi-radio multi-channel wireless networks. On the other aspect, several papers [6], [7] recently studied how to satisfy a certain traffic demand vector from all wireless nodes by a joint routing, link scheduling, and channel assignment under certain wireless interference models. Alicherry et al. [6] presented a linear programming based method to jointly perform multi-path routing, link scheduling, and static channel assignment for throughput optimization. $\mathrm{Li}$ et al. [8] also studied the throughput optimization via joint routing, link scheduling, and dynamic channel assignment for multi-radio multi-channel wireless networks. All these study either focused on the capacity of pure multi-hop mesh networks without gateways or assumed that the positions of mesh nodes and gateway nodes are fixed and given. In this paper, we consider the deployment of gateway nodes which affects the network throughput and capacity.

Deployment schemes of access points in WLAN has been studied [9]-[13] as well. However, most of the work focused on the guarantee of the coverage or how to provide better coverage using minimum number of access points. For example, in [13], Kouhbor et al. studied how to find the optimal number of access points and their locations for WLAN in an environment that includes obstacles. Notice that WLAN is different with WMN since WLAN only supports single-hop wireless communication while WMN is a multi-hop network. For multi-hop networks or hybrid networks, until recently there is only a few study on deployment of relay nodes or access points. Pabst et al. [14] showed that deployment of fixed relay nodes can enhance capacity in hybrid cellular networks. Fong et al. [15] also studied some fixed broadband wireless access deployment schemes to increase the network capacity. However, to the best of our knowledge, there is no previous study on how to deployment gateways in wireless mesh networks to maximize the throughput.

The rest of the paper is organized as follows. In Section II, we present our network model and interference model. We then mathematically formulate the throughput optimization problem for a fixed mesh network in Section III. An efficient gateway deployment scheme for throughput optimization is presented in Section IV. We discuss possible extensions of our proposed scheme in Section V. We report our simulation results that compare the performance of our methods with those of random and fixed methods in Section VI. We conclude our paper in Section VII.

\section{MODELS AND ASSUMPTIONS}

Network Model: A mesh network is modelled by a directed graph $G=(V, E)$, where $V=\left\{v_{1}, \ldots, v_{n}\right\}$ is the set of $n$ nodes and $E$ is the set of possible directed communication links. Let $\mathbf{E}^{-}(u)\left(\mathbf{E}^{+}(u)\right)$ denote the set of directed links that end (start) at node $u$. Every node $v_{i}$ has a transmission range $R_{T}(i):\left\|v_{i}-v_{j}\right\| \leq R_{T}(i)$ is not the sufficient condition for $\left(v_{i}, v_{j}\right) \in E$. Some links do not belong to $G$ because of either the physical barriers or the selection of routing protocols. We always use $\mathbf{L}_{i, j}$ to denote the directed link $\left(v_{i}, v_{j}\right)$ hereafter. For each link $e=(u, v)$, the maximum rate at which a mesh router $u$ can communicate with the mesh router $v$ in onehop communication supported by link $e$ is denoted by $\mathbf{c}(e)$. Notice that the links are directed, thus, the capacity could be asymmetric, i.e., $\mathbf{c}((u, v))$ may not same as $\mathbf{c}((v, u))$.

Among the set $V$ of all wireless nodes, some of them are gateways which have functionality and provide the connectivity to the Internet. For simplicity, let $\mathcal{S}=\left\{\mathbf{s}_{1}, \mathbf{s}_{2}, \cdots, \mathbf{s}_{k}\right\}$ be the set of $k$ gateway nodes, where $\mathbf{s}_{i}$ is actually node $v_{n+i-k}$, for $1 \leq i \leq k$. All other wireless nodes $v_{i}$ (for $1 \leq i \leq n-k$ ) $\in \overline{\mathcal{S}}=V-S$ are ordinary mesh nodes. Each ordinary mesh node $u$ will aggregate the traffic from all its users and then route them to the Internet through some gateway nodes. We assume that the capacity between any gateway node to the Internet is sufficiently large. We use $\ell_{O}(u)\left(\ell_{I}(u)\right)$ to denote the total aggregated outgoing (incoming) traffic for its users by mesh node $u$. We will mainly concentrate on one of the traffics in this paper, say incoming traffics. For notation simplicity, we use $\ell(u)$ to denote such load for node $u$. Notice that the traffic $\ell(u)$ is not requested to be routed through a specific gateway node, neither requested to be using a single routing path. Our results can be easily extended to deal with both incoming and outgoing traffic by defining routing flows for both traffics separately.

Interference Model: Each node $v_{i}$ also has an interference range $R_{I}(i)$ such that node $v_{j}$ is interfered by the signal from $v_{i}$ whenever $\left\|v_{i}-v_{j}\right\| \leq R_{I}(i)$ and $v_{j}$ is not the intended receiver. The interference range $R_{I}(i)$ is not necessarily same as the transmission range $R_{T}(i)$. Typically, $R_{T}(i)<R_{I}(i) \leq$ $c \cdot R_{T}(i)$ for some constant $c>1$. We call the ratio between them as the Interference-Transmission Ratio for node $v_{i}$, denoted as $\gamma_{i}=\frac{R_{I}(i)}{R_{T}(i)}$. In practice, $2 \leq \gamma_{i} \leq 4$. For all wireless nodes, let $\gamma=\max _{v_{i} \in V} \frac{R_{I}(i)}{R_{T}(i)}$.

To schedule two links at the same time slot, we must ensure that the schedule will avoid the link interference. Different types of link interference have been studied in the literature, such as Protocol Interferences Model(PrIM) [2], Fixed Protocol Interferences Model(fPrIM) [8], [16], RTS/CTS Model(RTS-CTS) [6], and Transmitter Interference Model(TxIM) [17]. In this paper we adopt the Fixed Protocol Interferences Model by assuming that any node $v_{j}$ will be interfered by the signal from $v_{k}$ if $\left\|v_{k}-v_{j}\right\| \leq R_{I}(k)$ and node $v_{k}$ is sending signal to some node other than $v_{j}$. In other words, the transmission from $v_{i}$ to $v_{j}$ is viewed successful if $\left\|v_{k}-v_{j}\right\|>R_{I}(k)$ for every node $v_{k}$ transmitting in the same time slot. Actually, our gateway deployment method can work for any kind of interference model as we will discuss in Section V. Given a network $G=(V, E)$, we use the conflict graph (e.g., [18]) $F_{G}$ to represent the interference in $G$. Each vertex (denoted by $\mathbf{L}_{i, j}$ ) of $F_{G}$ corresponds to a directed link 
$\left(v_{i}, v_{j}\right)$ in the communication graph $G$. There is a directed edge from vertex $\mathbf{L}_{i, j}$ to vertex $\mathbf{L}_{p, q}$ in $F_{G}$ if and only if the transmission of $\mathbf{L}_{i, j}$ interferes the reception of the receiving node of link $\mathbf{L}_{p, q}$.

\section{Throughput Optimization in Mesh Networks}

Wang et al. [16] recently studied the interference aware TDMA link scheduling when the load of each link is given for a static wireless mesh network. In this paper, we want to study what is the best throughput achievable by a given multi-hop mesh networks using best possible routing and link scheduling. We assume that the routing between a given mesh router and some gateway nodes could be using multiple paths. In practice, we do not need every session to be multi-path. We essentially assume that the aggregated traffics between the mesh router and the gateway nodes could be infinitely divisible. We also assume the time is slotted and synchronized.

Assume that every mesh router $u$ has a traffic demand $\ell(u)$ that needs to be routed to the Internet via some gateway nodes. We want to maximize the total routed traffic to the Internet while certain minimum traffic from each mesh router should be satisfied. Our approach is to give each link $\mathbf{L} \in G$ an interference-aware transmission schedule $\mathcal{S}(\mathbf{L})$ which assign the time slot for transmission to maximize the overall network throughout. A link scheduling is to assign each link a set of time slots $\subset[1, T]$ in which it will transmit, where $T$ is the scheduling period. A link scheduling is interference-aware (or called valid) if a scheduled transmission on a link $x \rightarrow y$ will not result in a collision at either node $x$ or node $y$ (or any other node) due to the simultaneous transmission of other links. Let $X_{e, t} \in\{0,1\}$ be the indicator variable which is 1 if and only if $e$ will transmit at time-slot $t$. We focus on periodic schedules here. A schedule is periodic with period $T$ if, for every link $e$ and time slot $t, X_{e, t}=X_{e, t+i \cdot T}$ for any integer $i \geq 0$. For a link $e$, let $I(e)$ denote the set of links $e^{\prime}$ that will cause interference if $e$ and $e^{\prime}$ are scheduled at the same time slot. A schedule $\mathcal{S}$ is interference-free if $X_{e, t}+X_{e^{\prime}, t} \leq 1, \forall e^{\prime} \in I(e)$.

We will provide a mixed Integer Programming formulation of the throughput optimization. For cross-layer optimization, the flow that can be supported by mesh networks not only need to satisfy the capacity constraint, but also need to be schedulable by all links without interference.

First, we formulate the routing problem to maximize the throughput of the achieved flow under certain fairness constraints. Let $\alpha(e) \in[0,1]$ denote the fraction of the time slots in one scheduling-period that link $e$ is actively transmitting. Obviously, $\alpha(e) \cdot \mathbf{c}(e)$ is the corresponding achieved flow. Given a routing (and corresponding link scheduling), the achieved fairness $\lambda$ is defined as the minimum ratio of achieved flow over the demanded load over all wireless mesh routers. Assume that we have a minimum fairness constraints $\lambda_{0}$. Clearly, the achieved flow at a router $u$ is difference between the flow goes out of node $u$ and the flow coming to node $u$, i.e., $\sum_{e \in \mathbf{E}^{+}(u)} f(e)-\sum_{e \in \mathbf{E}^{-}(u)} f(e)$. Here $f(e)$ is the total scheduled traffics over link $e$. The maximum throughput routing is equivalent to solve the following linear programming
(LP-Flow-throughput) for $\alpha(e, \mathbf{f})$ such that

\begin{tabular}{|c|c|c|}
\hline \multicolumn{3}{|c|}{ LP-Flow-throughput: $\max \sum_{i=1}^{k} f\left(\mathbf{s}_{i}\right)$} \\
\hline$\sum_{e \in \mathbf{E}^{+}(u)} f(e)-\sum_{e \in \mathbf{E}^{-}(u)} f(e)$ & $=f(u)$ & $\forall u \in \overline{\mathcal{S}}$ \\
\hline$f(u)$ & $\geq \lambda_{0} \ell(u)$ & $\forall u \in \overline{\mathcal{S}}$ \\
\hline$f(e)-\sum_{e \in \mathbf{E}^{+}\left(\mathbf{s}_{i}\right)} f(e)$ & $=f\left(\mathbf{s}_{i}\right)$ & $\forall \mathbf{s}_{i} \in \mathcal{S}$ \\
\hline$\alpha(e) \cdot \mathbf{c}(e)$ & $=f(e)$ & $\forall e$ \\
\hline$\alpha(e)$ & $\geq 0$ & $\forall e$ \\
\hline$\alpha(e)$ & $\leq 1$ & $\forall e$ \\
\hline sts interfence-free schedule for & $\alpha(e)$ & \\
\hline
\end{tabular}

Our objective of periodic TDMA link scheduling is to give each link $\mathbf{L} \in G$ a transmission schedule $\mathcal{S}(\mathbf{L})$, which is the list of time-slot that a link could send packets such that the schedule is interference-free. We then mathematically formulate a necessary, sufficient condition for schedulable flow $f(e)=\alpha(e) \cdot \mathbf{c}(e)$ : a flow $f$ (equivalently, whether a given vector $\alpha(e)$ for all $e$ is schedulable) is schedulable iff we can find integer solution $X_{e, t}$ satisfying the following conditions.

Necessary and Sufficient Condition for Schedulable Flow:
\[ \left\{\begin{array}{rll}X_{e, t}+X_{e^{\prime}, t} & \leq 1 & \forall e^{\prime} \in \mathbf{I}(e), \forall e, \forall t \\ \frac{\sum_{1 \leq t \leq T} X_{e, t}}{T} & =\alpha(e) \quad \forall e \\ X_{e, t} & \in\{0,1\} \quad \forall e, \forall t\end{array}\right. \]

The first condition says that a schedule should be interferencefree. The second condition says the the schedule should achieve the required flow $\alpha(e)$. It is widely known that it is NP-hard to decide whether a feasible scheduling $X_{e, t}$ exists when given the flow $f(e)$ (or equivalently, $\alpha(e)$ ) for wireless networks with interference constraints. For some interference models several papers gave relaxed necessary conditions and relaxed sufficient conditions for schedulable flows that can be decided in polynomial time. For example, in [8] proved a necessary and a sufficient condition for schedulable flows under different interference model. Consider the active fraction $\alpha(e) \in[0,1]$ of each link. A sufficient condition that this $\alpha$ is schedulable is, for each $\alpha(e)+\sum_{e^{\prime} \in \mathbf{I}_{\mathcal{M}}(e)} \alpha\left(e^{\prime}\right) \leq 1$. A necessary condition that this $\alpha$ is schedulable is, for each $\alpha(e)+\sum_{e^{\prime} \in \mathbf{I}_{\mathcal{M}}(e)} \alpha\left(e^{\prime}\right) \leq C_{\mathcal{M}}$. Here $\mathbf{I}_{\mathcal{M}}(e) \subseteq \mathbf{I}(e)$ is defined based on the specific interference model $\mathcal{M}$ for the purpose of link scheduling; $C_{\mathcal{M}}$ is a constant depending on the specific interference model and $\gamma$. For the Fixed Protocol Interferences Model, $C_{\mathcal{M}}=\left\lceil\frac{2 \pi}{\arcsin \frac{\gamma-1}{2 \gamma}}\right\rceil$, e.g., $C_{\mathcal{M}}=25$ when $\gamma=2$ [8]. Then we can relax the original mixed Integer Programming to a linear programming by getting rid of the scheduling variables $X$. Based on previous study, we generally require that, given a constant integer $C \in\left[1, C_{\mathcal{M}}\right]$, we need to solve the following Linear Programming (LP-Flow-Throughput) for $\alpha(e)$ such 
that

$$
\begin{aligned}
& \text { LP-Flow-throughput: } \max \sum_{i=1}^{k} f\left(\mathbf{s}_{i}\right) \\
& \left\{\begin{aligned}
\sum_{e \in \mathbf{E}^{+}(u)} f(e)-\sum_{e \in \mathbf{E}^{-}(u)} f(e) & =f(u) & & \forall u \in \overline{\mathcal{S}} \\
f(u) & \geq \lambda_{0} \ell(u) & & \forall u \in \overline{\mathcal{S}} \\
\sum_{e \in \mathbf{E}^{-}\left(\mathbf{s}_{i}\right)} f(e)-\sum_{e \in \mathbf{E}^{+}\left(\mathbf{s}_{i}\right)} f(e) & =f\left(\mathbf{s}_{i}\right) & & \forall \mathbf{s}_{i} \in \mathcal{S} \\
\alpha(e) \cdot \mathbf{c}(e) & =f(e) & & \forall e \\
\alpha(e) & \geq 0 & & \forall e \\
\alpha(e) & \leq 1 & & \forall e \\
\alpha(e)+\sum_{e^{\prime} \in \mathbf{I}_{\mathcal{M}}(e)} \alpha\left(e^{\prime}\right) & \leq C & & \forall e
\end{aligned}\right.
\end{aligned}
$$

In [16], Wang et al. studied ink scheduling for networks of single channel. We can apply their greed method to design efficient link scheduling that can achieve $\alpha(e)$ found from the solution of the LP. Assume that we already have the values $\alpha(e)$ for every links $e$ and $T$ is the number of time slots per scheduling period. Then we need to schedule $T \cdot \alpha(e)$ time-slots for a link $e$. For simplicity, we assume that the chosen of $T$ results that $T \cdot \alpha(e)$ is integer for every $e$. Notice that when we schedule each link, we need to ensure that the scheduling is interference-free. Algorithm 1 illustrates our scheduling method. The basic idea of our scheduling is first sorting the links based on some specific order and then process the requirement $\alpha(e)$ for each link in a greedy manner.

\section{Algorithm 1 Greedy Link Scheduling}

Input: A communication graph $G=(V, E)$ of $m$ links and $\alpha(e)$ for all links.

Output: An interference-free link scheduling.

1: Sort the links in the communication graph $G$ according the interference model. For fPrIM model, we consider the conflict graph. We choose the vertex, which is the link in the original graph, with the largest value $d_{i, j}^{i n}-d_{i, j}^{\text {out }}$ in the residue conflict graph; remove the vertex and its incident edges. Where $d_{i, j}^{i n}$ and $d_{i, j}^{\text {out }}$ are the in-degree and out-degree of vertex $\mathbf{L}_{i, j}$ in the conflict graph for the fPrIM interference model. Repeat this process until there is no vertex in the graph. Then the links (in the original graph) are sorted by their reverse removal order. Let $\left(e_{1}, e_{2}, \cdots, e_{m}\right)$ be the sorted list of links.

2: for $i=1$ to $m$ do

3: $\quad N\left(e_{i}\right)=T \cdot \alpha\left(e_{i}\right)$ be the number of time slots that link $e_{i}$ will be active.

4: $\quad$ Assume $e_{i}=(u, v)$. Set allocated $\leftarrow 0 ; t \leftarrow 1$;

5: $\quad$ while allocated $<N(e)$ do

6: $\quad$ if $X_{e^{\prime}, t}=0$ for every conflicting link $e^{\prime} \in \mathbf{I}_{\mathcal{M}}\left(e_{i}\right)$, $\sum_{e^{\prime}: e^{\prime} \ni u} X_{e^{\prime}, t}<1, \sum_{e^{\prime}: e^{\prime} \ni v} X_{e^{\prime}, t}<1$ then

7: $\quad$ Set $X_{e_{i}, t} \leftarrow 1$; Set allocated $\leftarrow$ allocated +1 ;

8: $\quad$ Set $t \leftarrow t+1$.

Notice that our algorithm relies on some special sorting of the links, depending on the interference models. We process links in this sorted order. When process the $i^{\text {th }}$ link $e_{i}$, we assign link $e_{i}$ the earliest (not need to be consecutive) $N\left(e_{i}\right)=T \cdot \alpha\left(e_{i}\right)$ time slots that will not cause any interference to already scheduled links. In [8], the authors proved that Algorithm 1 produces a feasible interference-free link scheduling when $\alpha(e)$ is a feasible solution of LP using $C=1$.

\section{Gateways Placement Schemes}

Last section provide a method (Algorithm 1 together with the linear programming formulation LP-Flow-Throughput) produces a feasible interference-free link scheduling which maximize the network throughput. In other words, this method can be used to evaluate a fixed mesh networks with certain gateways in term of throughput optimization. In this section, we propose a grid-based gateway placement scheme which uses the linear programming LP-Flow-Throughput as a evaluation tool. The problem we want to study is as follows:

The Problem: Given a mesh network with $n-k$ fixed mesh nodes and inference model, our gateway placement need to select positions for $k$ gateways in order to maximize the throughput. It is clear that we can not try all possible positions since the possible combination is infinite.

Random Deployment: The easiest and simplest method is random deployment where we randomly select $k$ positions for gateways. For example, Figure 2(a) shows 4 gateways are deployed randomly in a mesh network. However, the random deployment maybe not good at the throughput or even can not guarantee the connectivity of the mesh network.

Fixed Deployment: The second method is to deployment the gateways in fixed positions which are the centers of evenly distributed cells. As shown in Figure 2(b), to place 4 gateways, we divide the whole area into 4 cells and put the gateways in the centers of these cells. This fixed deployment scheme should be able to work well with well-spread and evenlydistributed mesh networks. However, if the network is not so even, for example, putting a gateway at the center of the leftupper cell in Figure 2(b) does not help a lot for the throughput since the gateway can only connect 2 mesh nodes and one of them is an end-point.

Grid Based Deployment: To explore more choices of gateway layout but in the same time to keep the scalability of the proposed method, we propose a new grid based deployment scheme. The idea is simple. We first divide the whole deployment area into a $a \times b$ grid. As shown in Figure 2(c), we only place the gateways in the cross points on this grid. We will try all possible combinations of the $k$-gateway placement, and evaluate each of them using the method in previous section by computing the maximum throughput can be achieved by this combination. For a $a \times b$ grid, the number of total combinations is $C_{a \times b}^{k}$ which is the combination of selecting $k$ elements from $a \times b$ elements. Even though this number could be large, it is still reasonable to try all of them since the deployment scheme will only run once before the real gateway installation and positions of all the mesh routers are fixed. In addition, the number of overhead is depended on the size of the grid. It is an adjustable parament which can be easily controlled for the tradeoff between computation overhead and throughput performance. If both $a$ and $b$ go to infinite, our grid-based method can explore all possible deployment layout. 


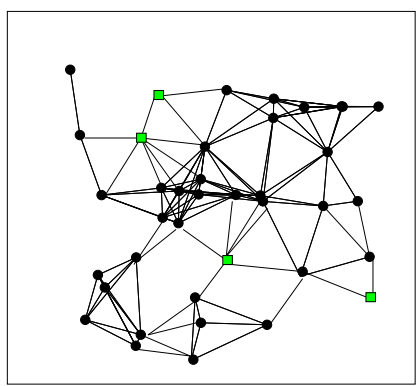

(a) Random Deployment

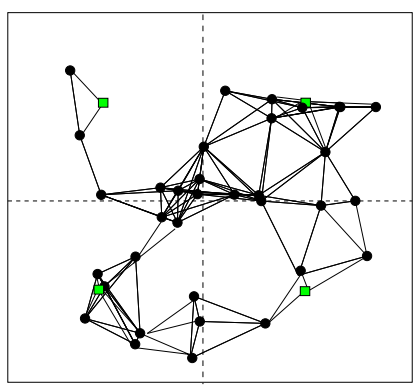

(b) Fixed Deployment

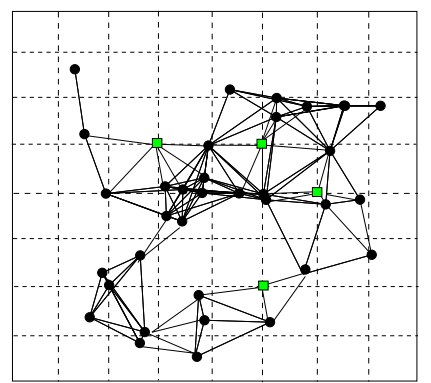

(c) Grid-based Deployment

Fig. 2. Three gateway deployment methods: 4 gateways (grey squre) are deployed in a mesh network with 33 mesh nodes (black dot).

We will test all these three methods by conducing simulations with random networks in Section VI.

\section{Discussions}

So far, we only consider the network with a single channel and using the fPrIM. However, our gateway placement method based on throughput optimization can be extended for various networks with different models.

Various Interference Models: Our maximum throughput method can be extended to deal with different interference models, such as PrIM [2], RTS-CTS [6], and TxIM [17]. The differences of these models with the fPrIM are that they have different definitions of link interference for two links. The only places in our method need to change are (1) the sorting method in Step 1 of Algorithm 1; (2) the constant $C \mathcal{M}$ with respect to all interference models $\mathcal{M}$. In [8], the authors showed how to do the sorting for different interference models and provided the values of $C_{\mathcal{M}}$ for those models.

Multi-channel and Multi-radio Networks: A number of schemes [19]-[22] have been proposed recently to exploit multiple channels and multiple radios for performance improvement in wireless mesh networks. Using multiple channels and multiple radios can alleviate but not eliminate the interference. Let $\mathcal{F}$ be the set of orthogonal channels that can be used by all wireless nodes. Each wireless terminal $u$ is equipped with $\mathcal{I}(u) \geq 1$ radio interfaces. However, here we assume that each wireless node $u$ can only operate on a subset of channels $\mathcal{F}(u)$ from $\mathcal{F}$ due to the hardware constraints. For notational convenience, we use $\mathcal{F}(e)$ to denote the set of common channels among $\mathcal{F}(u)$ and $\mathcal{F}(v)$ for any link $e=(u, v)$, and $\delta(e, \mathbf{f}) \in\{0,1\}$ be the indicator function whether a channel $\mathbf{f}$ can be used by a link $e$. For each link $e=(u, v)$ operating on a channel $\mathbf{f} \in \mathcal{F}(e)$, we denote by $\mathbf{c}(e, \mathbf{f})$ the rate for link $e$. This is the maximum rate at which a mesh router $u$ can communicate with the mesh router $v$ in one-hop communication using channel f. Let $\alpha(e, \mathbf{f}) \in[0,1]$ denote the fraction of the time slots in one scheduling-period that link $e$ is actively transmitting using channel f. Obviously, $\alpha(e, \mathbf{f}) \cdot \mathbf{c}(e, \mathbf{f})$ is the corresponding achieved flow.

For multi-channel and multi-radio mesh networks, we can refine our linear programming for throughput optimization as follows.

$$
\begin{aligned}
& \text { LP-Flow-throughput: } \max \sum_{i=1}^{k} f\left(\mathbf{s}_{i}\right) \\
& \left\{\begin{array}{rlrl}
\sum_{e \in \mathbf{E}^{+}(u)} f(e)-\sum_{e \in \mathbf{E}^{-}(u)} f(e) & =f(u) & & \forall u \in \overline{\mathcal{S}} \\
f(u) & \geq \lambda_{0} \ell(u) & \forall u \in \mathcal{\mathcal { S }} \\
\sum_{e \in \mathbf{E}^{-}\left(\mathbf{s}_{i}\right)} f(e)-\sum_{e \in \mathbf{E}^{+}\left(\mathbf{s}_{i}\right)} f(e) & =f\left(\mathbf{s}_{i}\right) & & \forall \mathbf{s}_{i} \in \mathcal{S} \\
\sum_{\mathbf{f} \in \mathcal{F}(e)} \alpha(e, \mathbf{f}) \cdot \mathbf{c}(e, \mathbf{f}) & =f(e) & & \forall e \\
\alpha(e, \mathbf{f}) & \geq 0 & & \forall e \\
\alpha(e, \mathbf{f}) & \leq 1 & & \forall e \\
\alpha(e, \mathbf{f})+\sum_{e^{\prime} \in \mathbf{I}_{\mathcal{M}}(e)} & \leq C & \forall e \\
\alpha(e, \mathbf{f}) & \leq \delta(e, \mathbf{f}) & \\
\sum_{e \ni u, \mathbf{f}} \alpha(e, \mathbf{f}) & \leq \mathcal{I}(u) &
\end{array}\right.
\end{aligned}
$$

The link scheduling in multi-channel and multi-radio mesh networks also need satisfy the channel and radio constraints no matter whether dynamic channel assignment or fixed channel assignment is used. The greedy link scheduling will become as follows.

Algorithm 2 Greedy Link Scheduling for Multi-Radio MultiChannel Networks

Input: A communication graph $G=(V, E)$ of $m$ links and $\alpha(e, \mathbf{f})$ for all links and channels.

Output: An interference-free link scheduling.

1: Sort the links in the communication graph $G$ as the same in Algorithm 1. Let $\left(e_{1}, e_{2}, \cdots, e_{m}\right)$ be the sorted list of links.

2: for $i=1$ to $m$ do

3: for each possible channel $\mathbf{f} \in \mathcal{F}$ do

4: $\quad$ Let $N\left(e_{i}, \mathbf{f}\right)=T \cdot \alpha\left(e_{i}, \mathbf{f}\right)$ be the number of time slots that link $e_{i}$ will be active using channel $\mathbf{f}$.

5: $\quad$ Assume $e_{i}=(u, v)$. Set allocated $\leftarrow 0 ; t \leftarrow 1$;

6: $\quad$ while allocated $<N(e, \mathbf{f})$ do

7: if $X_{e^{\prime}, t, \mathbf{f}}=0$ for every conflicting link $e^{\prime} \in \mathbf{I}_{\mathcal{M}}\left(e_{i}\right), \quad \sum_{\mathbf{f}, e^{\prime}: e^{\prime} \ni u} X_{e^{\prime}, t, \mathbf{f}}<\mathcal{I}(u)$, $\sum_{\mathbf{f}, e^{\prime}: e^{\prime} \ni v} X_{e^{\prime}, t, \mathbf{f}}<\mathcal{I}(v)$ then

8: $\quad$ Set $X_{e_{i}, t, \mathbf{f}} \leftarrow 1$; Set allocated $\leftarrow$ allocated +1 ; 9: $\quad$ Set $t \leftarrow t+1$.

Notice that when process the $i$ th link $e_{i}$, we process the channels in order and assign link $e_{i}$ the earliest (not need to be consecutive) $N\left(e_{i}, \mathbf{f}\right)=T \cdot \alpha\left(e_{i}, \mathbf{f}\right)$ time slots using channel $\mathbf{f}$ 
that will not cause any interference to already scheduled links, and satisfies the radio and channel-availability constraints.

\section{Simulations}

In this section, we exam the maximal flow of different gateway deployment schemes in random wireless mesh networks. As we have discussed in Section III, the maximal flow is solved by a linear program. The wireless mesh network in this paper is generated randomly where the position of $n$ mesh nodes is chosen randomly. Then the deployment method will decide to place $k$ gateways to connect the mesh routers to Internet. We use $802.11 a$ for the link channel capacity in the wireless mesh network, which is same as [6]. The link channel capacity thus only depends on the distance between the two nodes at the end of the link. We set the link channel capacity as $54 M$ bps when the distance of the two end nodes is within 30 meters, $48 M b p s$ when the distance is within 32 meters, $36 M b p s$ when the distance is within 37 meters, $24 M b p s$ when the distance is within 45 meters, $18 M b p s$ when the distance is within 60 meters, $12 \mathrm{Mbps}$ when the distance is within 69 meters, $9 M b p s$ when the distance is within 77 meters, and $6 M b p s$ when the distance is within 90 meters. Otherwise, if the distance of the two end nodes of the link is beyond 90 meters, we will set the link channel capacity as 0. Each node has 180 meters interference range. The wireless mesh network is generated with $60-100$ mesh routers and $4-8$ gateways. The mesh routers are randomly dispersed in a square area of $500 \times 500$ square meters. Each mesh router transfers $20 \mathrm{Mbps}$ data to the Internet. The input value of $\lambda_{0}$ and $C$ in the LP to solve the maximal throughput is set as 0.2 and 20 .

We exam three gateway deployment schemes described in Section IV. The fixed deployment scheme first divides the square area into $k$ equal cells as shown in Figure 3(a)-(c), and then put the $k$ gateways in the centers of these cells. Our gridbased deployment scheme will use various grids defined in Figure 3(d)-(e) to define the candidate positions of gateways, and then try all the combinations of positions using the LP to evaluate their throughput, and select the combination with highest throughput.

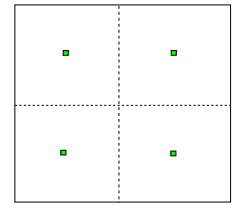

(a) 4 Gateways

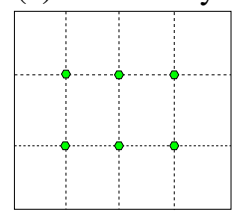

(d) $2 \times 3$ Grid

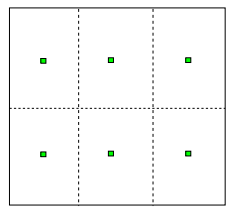

(b) 6 Gateways

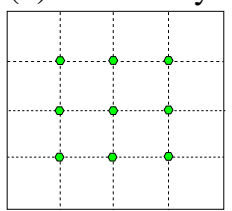

(e) $3 \times 3$ Grid

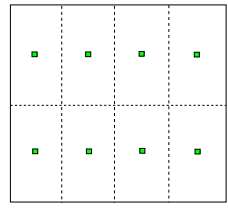

(c) 8 Gateways

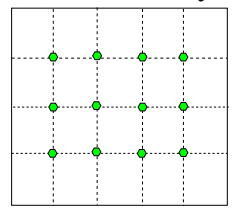

(f) $3 \times 4$ Grid
Fig. 3. The layouts of gateways in fixed deployment scheme (a-c) and the grids used in our deployment scheme (d-f).

We various the numbers of mesh routers, mesh gateways and cells of the grid to test the performance of these three deployment schemes.

Table VI shows the results for networks with 60,80 and 100 mesh routers and 6 gateways to be deployed. It is clear that our grid-based method can achieve better throughput than the random and fixed schemes. Notice that there are cases that the random deployment method can not find feasible solutions in LP or even can not form a connected mesh network. We exclude those cases in the results present here. In other words, all the data here are for the mesh network where the random deployment can find the feasible solution. For results in Table VI, the throughput increases when the network has more mesh routers.

Table VI shows the results when we want to deploy various number of gateways. The number of gateways is from 4 to 8 when the number of mesh routers are fixed at 65 . It is clear that with more gateways the performance is better.

Table VI shows the results when we increasing the size of the grid from $2 \times 3$ to $3 \times 4$ when the number of gateway fixed at 6 . It is clear that the larger size of grid can improves the throughput, but also increases the computation cost. Therefore, in practice, the administrator need to find an appropriate grid to satisfy both performance and cost requirements. On the other hand, by having the ability to change the grid size, it gives the way for administrator to play the tradeoff.

TABLE I

AVG THROUGHPUT ACHIEVED BY DIFFERENT DEPLOYMENT SCHEMES (WITH VARIOUS NETWORK SIZE)

\begin{tabular}{|c|c|l|l|l|}
\hline Nodes & Gateways & Random & Fixed & $3 \times 4$ Grid \\
\hline 60 & 6 & 579.75 & 582.07 & 582.17 \\
80 & 6 & 865.95 & 776.93 & 866.53 \\
100 & 6 & 804.91 & 810.02 & 813.50 \\
\hline
\end{tabular}

TABLE II

AVG THROUGHPUT ACHIEVED BY DIFFERENT DEPLOYMENT SCHEMES (WITH VARIOUS NUMBER OF GATEWAYS)

\begin{tabular}{|c|c|l|l|l|}
\hline Nodes & Gateways & Random* & Fixed & $3 \times 4$ Grid \\
\hline 65 & 4 & 384.84 & 384.39 & 392.80 \\
65 & 6 & 476.71 & 476.76 & 477.93 \\
65 & 8 & 843.44 & 843.37 & 843.44 \\
\hline
\end{tabular}

TABLE III

AVG THROUGHPUT ACHIEVED BY DIFFERENT DEPLOYMENT SCHEMES (WITH VARIOUS GRID SIZE FOR OUR SCHEME)

\begin{tabular}{|c|c|l|l|l|}
\hline Nodes & Gateways & $2 \times 3$ Grid & $3 \times 3$ Grid & $3 \times 4$ Grid \\
\hline 65 & 6 & 815.58 & 824.97 & 825.67 \\
80 & 6 & 776.93 & 866.53 & 866.53 \\
100 & 6 & 810.02 & 811.07 & 813.50 \\
\hline
\end{tabular}

\section{CONCLUSION}

\section{REFERENCES}

[1] X. W. Ian F. Akyildiz and W. Wang, "Wireless mesh networks: a survey," Computer Networks, vol. 47, no. 4, pp. 445-487, 2005. 
[2] P. Gupta and P. Kumar, "Capacity of wireless networks," University of Illinois, Urbana-Champaign, Tech. Rep., 1999.

[3] J. Li, C. Blake, D. S. D. Couto, H. I. Lee, and R. Morris, "Capacity of ad hoc wireless networks,' in MobiCom '01: Proceedings of the 7th annual international conference on Mobile computing and networking. New York, NY, USA: ACM Press, 2001, pp. 61-69.

[4] M. Grossglauser and D. Tse, "Mobility increases the capacity of ad-hoc wireless networks," in INFOCOMM, vol. 3, 2001, pp. $1360-1369$. [Online]. Available: citeseer.nj.nec.com/499040.html

[5] P. Kyasanur and N. H. Vaidya, "Capacity of multi-channel wireless networks: impact of number of channels and interfaces," in MobiCom '05: Proceedings of the 11th annual international conference on Mobile computing and networking. New York, NY, USA: ACM Press, 2005, pp. 43-57.

[6] M. Alicherry, R. Bhatia, and L. E. Li, "Joint channel assignment and routing for throughput optimization in multi-radio wireless mesh networks," in MobiCom '05: Proceedings of the 11th annual international conference on Mobile computing and networking. New York, NY, USA: ACM Press, 2005, pp. 58-72.

[7] M. Kodialam and T. Nandagopal, "Characterizing achievable rates in multi-hop wireless networks: the joint routing and scheduling problem," in MobiCom '03: Proceedings of the 9th annual international conference on Mobile computing and networking. New York, NY, USA: ACM Press, 2003, pp. 42-54.

[8] X.-Y. Li, Y. Wu, and W. Wang, "Throughput optimization in multi-hop multi-radio multi-channel wireless networks," 2006.

[9] K. Maksuriwong, V. Varavithya, and N. Chaiyaratana, "Wireless lan access point placement using multiobjective genetic algorithm," in Proceedings of IEEE International Conference on Systems, Man \& Cybernetics, 2003.

[10] S. Kouhbor, J. Ugon, A. Rubinov, A. Kruger, and M. Mammadov, "Wlan coverage planning: Optimization models and algorithms," in Proceedings of the first IEEE International Conference on Wireless Broadband and Ultra Wideband Communications, 2006.

[11] R. Rodrigues, G. Mateus, and A. Loureiro, "Optimal base station placement and fixed channel assignment applied to wireless local area network projects," in ICON '99: Proceedings of the 7th IEEE International Conference on Networks. Washington, DC, USA: IEEE Computer Society, 1999, p. 186.

[12] G. de la Roche, R. Rebeyrotte, K. Jaffres-Runser, and J.-M. Gorce, "A qos-based fap criterion for indoor 802.11 wireless lan optimization," in Proceedings of IEEE International Conference on Communications (ICC2006), 2006.

[13] S. Kouhbor, J. Ugon, A. Rubinov, A. Kruger, and M. Mammadov, "Coverage in wlan with minimum number of access points," in Proceedings of IEEE 63rd Vehicular Technology Conference, 2006. VTC 2006-Spring, 2006.

[14] R. Pabst, B. Walke, D. Schultz, P. Herhold, H. Yanikomeroglu, S. Mukherjee, H. Viswanathan, M. Lott, W. Zirwas, M. Dohler, H. Aghvami, D. Falconer, and G. Fettweis, "Relay-based deployment concepts for wireless and mobile broadband radio," IEEE Communications Magazine, vol. 42, no. 9, pp. 80-89, 2004

[15] B. Fong, N. Ansari, A. Fong, G. Hong, and P. Rapajic, "On the scalability of fixed broadband wireless access network deployment," IEEE Communications Magazine, vol. 42, no. 9, pp. 12-18, 2004.

[16] W. Wang, Y. Wang, X.-Y. Li, W.-Z. Song, and O. Frieder, "Efficient interference-aware tdma link scheduling for static wireless networks," in 12th ACM Annual International Conference on Mobile Computing and Networking (MobiCom 2006), 2006.

[17] S. Yi, Y. Pei, and S. Kalyanaraman, "On the capacity improvement of ad hoc wireless networks using directional antennas," in Proceedings of the 4th ACM MobiHoc, 2003, pp. 108-116.

[18] K. Jain, J. Padhye, V. N. Padmanabhan, and L. Qiu, "Impact of interference on multi-hop wireless network performance," in MobiCom '03: Proceedings of the 9th annual international conference on Mobile computing and networking. New York, NY, USA: ACM Press, 2003, pp. 66-80.

[19] A. Raniwala and T. Chiueh, "Architecture and algorithms for an ieee 802.11-based multi-channel wireless mesh network," in INFOCOM 2005.

[20] P. Kyasanur and N. H. Vaidya, "Routing and interface assignment in multi-channel multi-interface wireless networks," in WCNC 2005.

[21] R. Draves, J. Padhye, and B. Zill, "Routing in multi-radio, multi-hop wireless mesh networks," in ACM Mobicom 2004.
[22] J. Wang, Y. Fang, and D. Wu, "A power-saving multi-radio multi-channel mac protocol for wireless local area networks," in in IEEE International Conference on Computer Communication (INFOCOM'06), 2006. 